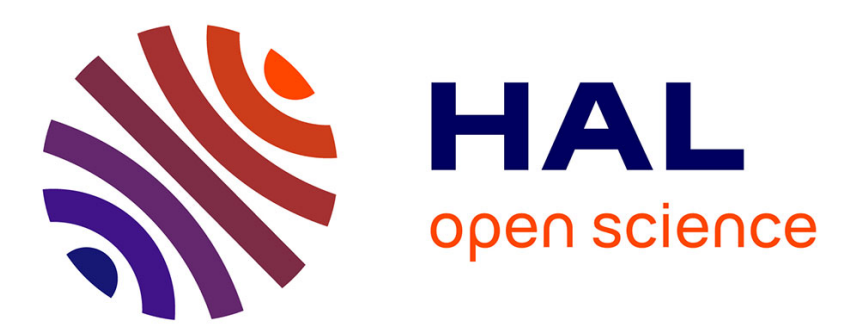

\title{
Morphological Texture Description from Multispectral Skin Images in Cosmetology
}

Joris Corvo, Jesus Angulo, Josselin Breugnot, Sylvie Bordes, Brigitte Closs

\section{To cite this version:}

Joris Corvo, Jesus Angulo, Josselin Breugnot, Sylvie Bordes, Brigitte Closs. Morphological Texture Description from Multispectral Skin Images in Cosmetology. 13th International Symposium on Mathematical Morphology and Its Applications to Signal and Image Processing, May 2017, Fontainebleau, France. pp.443 - 477, 10.1007/978-3-319-57240-6_38. hal-01536371

\section{HAL Id: hal-01536371 \\ https: / hal-mines-paristech.archives-ouvertes.fr/hal-01536371}

Submitted on 11 Jun 2017

HAL is a multi-disciplinary open access archive for the deposit and dissemination of scientific research documents, whether they are published or not. The documents may come from teaching and research institutions in France or abroad, or from public or private research centers.
L'archive ouverte pluridisciplinaire HAL, est destinée au dépôt et à la diffusion de documents scientifiques de niveau recherche, publiés ou non, émanant des établissements d'enseignement et de recherche français ou étrangers, des laboratoires publics ou privés. 


\title{
Morphological Texture Description from Multispectral Skin Images in Cosmetology
}

\author{
Joris Corvo $^{1}$, Jesus Angulo ${ }^{2}$, Josselin Breugnot ${ }^{1}$, Sylvie Bordes ${ }^{1}$, and Brigitte Closs ${ }^{1}$ \\ 1 SILAB, BP 213, 19108 Brive Cedex, France. \\ $\{j$.corvo, j.breugnot, s.bordes, b.closs\}@silab.fr \\ 2 Center for Mathematical Morphology, Mines-ParisTech, \\ PSL Research University, 77300 Fontainebleau, France. \\ jesus.angulo@mines-paristech.fr
}

\begin{abstract}
In this paper, we propose methods to extract texture features from multispectral skin images. We first describe the acquisition protocol and corrections we applied on multispectral skin images. In the framework of a cosmetology application, a skin morphological texture evaluation is then proposed using either multivariate approach on multispectral dataset or marginal on a dataset whose dimensionality has been reduced by a multivariate analysis based on PCA.
\end{abstract}

Keywords: Multispectral imaging, mathematical morphology, texture analysis, cosmetology.

\section{Introduction}

Most common image texture features have been primarily designed for 2D or 3D scalar images. Those features focus on variations in the spatial domain living up information contained in spectral domain. However, in some classification studies [10 25] the definition of color or multispectral texture features led to better results than classical scalar texture features. This study tackles the issue of multispectral texture extraction in the context of skin description and classification.

In literature, three main approaches are used to obtain texture features from multichromatic images [12/25]:

- In the parallel approach, texture and spectral data are considered separately. In other words, spatial information is extracted on a greyscale version of initial images, whereas spectrum (or color) information is measured globally. This approach is often used in image retrieval [24].

- Frequently used in material analysis [1930], the sequential approach consists in labeling the image spectra before extracting texture features. Usual scalar texture algorithms are processed on the labeled imaged since each label value can be considered as a scalar. The major disadvantage of this method is its lack of reproducibility induced by the preliminary step of image labeling. 
- The integrative approach regroups two alternatives for multivariate image texture analysis. The first one is the marginal integrative approach. It proposes to compute usual scalar texture features on each image component separately [10|27]. On the contrary, the second alternative is called multivariate integrative approach and relies on different algorithms allowing to extract texture features using all image components simultaneously [1|15|16|26|25|31|34].

In this paper, we adopt the integrative approach since we intend to define multispectral texture descriptors which are not content-dependent. In particular, we focus on building up multispectral texture features corresponding to the multivariate integrative approach, i.e., texture features for which every single value is obtained using all available wavelengths of a given multispectral image.

The new multispectral texture descriptors are applied to a database of human skin multispectral images acquired in-vivo within the context of a cosmetological study. Texture data are employed to analyze and predict the degradation of a foundation make-up. In the next section, we briefly introduce the database acquisition protocol and the different preprocessing steps applied to each image. Section 2 presents an original multivariate analysis called Inverted and Permuted Principal Component Analysis (IP-PCA)[9], used to reduce image dimensionality by projecting images components into consistent orthogonal spaces of representation. All multispectral texture parameters proposed in this paper are detailed in Sect.4. Finally, in Sect. 5 we use data from the cosmetological study to illustrate and assess the multispectral texture efficiency.

\section{Multispectral image acquisition and preprocessing}

\subsection{Acquisition device}

Our base of skin images was obtained using a multispectral camera called Asclepios System. This camera designed by the Le2i Laboratory was originally dedicated to dermatological issues [17|18]35]. Inside the Asclepios System, light emitted by a constant light source passes through a wheel of 10 spectral filters and is guided to a hand-held acquisition device directly placed on the volunteers skin. The device contains a greylevel CCD camera synchronized to the filter wheel so that it acquires reflection images whose illuminant only depends on single spectral filter of the wheel. Functional schema of Fig. 1 gives an overview of the system different components.

Each multispectral acquisition is taken in about $2.5 \mathrm{~s}$ and is composed of 10 gray level spectral images between $430 \mathrm{~nm}$ and $85 \mathrm{~nm}$. A raw acquisition is illustrated in Fig. 2. Spectral images are sized $1312 \times 1082$ pixels for approximately $35 \mathrm{~mm} \times 28 \mathrm{~mm}$ surface of skin $\left(2,67 \times 10^{-2} \mathrm{~mm}\right.$ par pixel). This definition has proved to be sufficient to observe the impact of make-up on skin texture. 


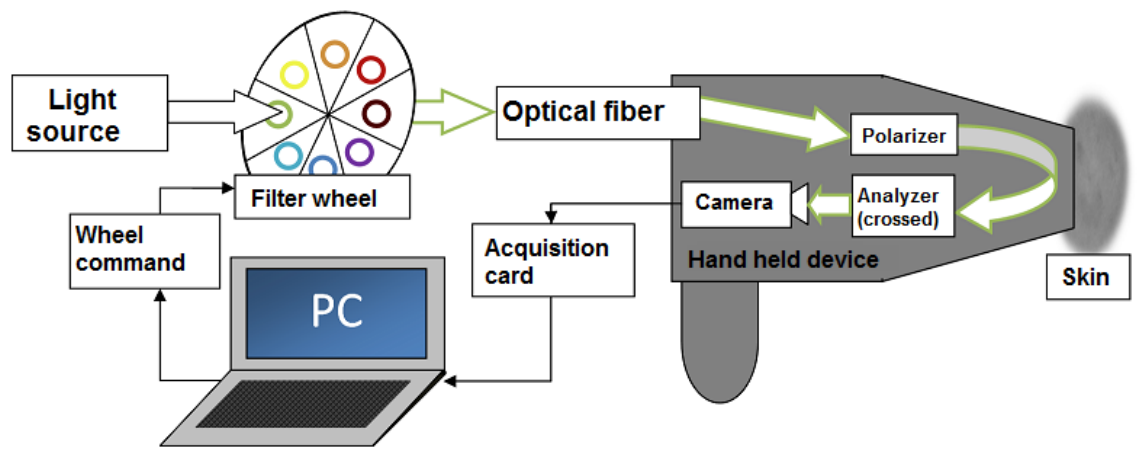

Fig. 1. Functional schema of the Asclepios System.

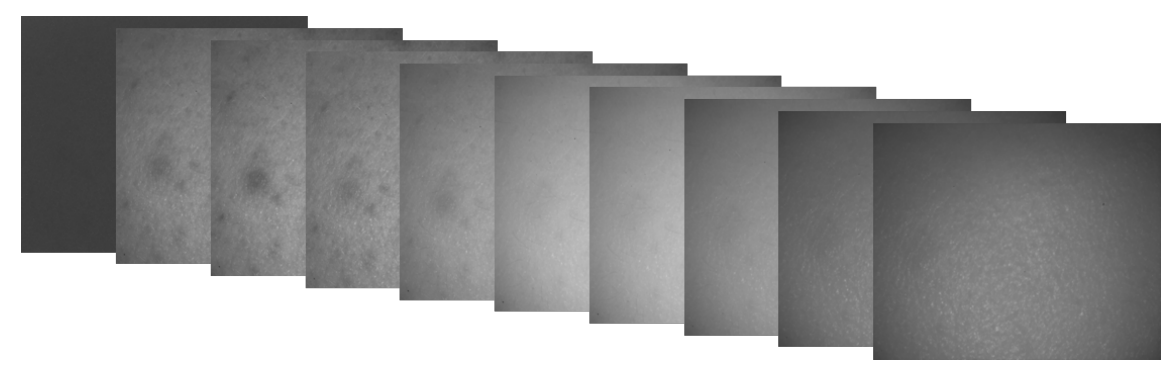

Fig. 2. Example of skin image raw acquisition from Asclepios System. 


\subsection{Cosmetology protocol for database generation}

The study of foundation make up effect and degradation on multispectral images skin was led on a panel of 30 volunteers. All of them applied the same amount of foundation make up on their faces. Multispectral acquisitions were taken on their cheek. Only left side of their faces was made up so that the other side could serve as normalization value. In order to track in time the make-up degradation, 4 acquisitions series were realized on each volunteer. The two firsts corresponding to the skin before and right after application, whereas the two lasts were taken respectively 3 and 6 hours after application. An example of image time series is given in Fig. 3. We note that for the purpose of visualization, a RGB color rendering from the multispectral is used. This rendering is based on pixel-wise linear regressions that associate RGB values to each pixel expressed in the Asclepios spectral space. Those regressions are estimated using several Asclepios multispectral acquisitions of a Macbeth colorchart.

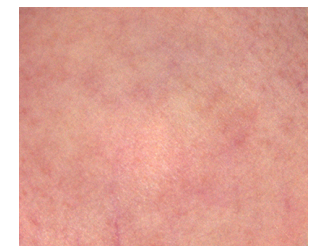

(a) Before application

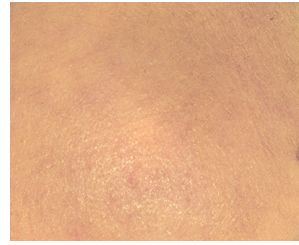

(b) After application

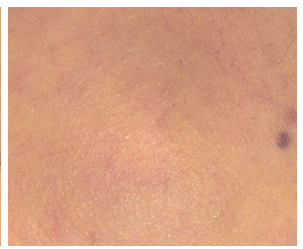

(c) 3 hours later

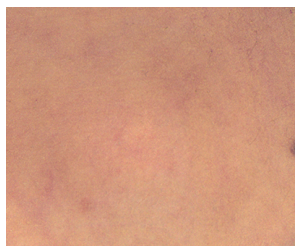

(d) 6 hours later

Fig. 3. Color-rendering of Asclepios system acquisitions of the same volunteer on the left cheek at four different times.

\subsection{Image prepocessing}

Images acquired through the Asclepios system suffer from various perturbations leading to degraded images. In order to increase the quality of computed texture features, we seek to improve collected data by correcting and standardizing the multispectral raw images. A chain of four different preprocessing steps is applied to the data set in a specific order.

First of all, image normalization is performed by subtracting from each spectral image the average spectral image of the same wavelength. This results in eliminating the illumination gradient and some constant artifacts, e.g., Fig. 4(b)

Then a Vector Median Filtering (VMF) [2] is implemented to filter out noise of multispectral images without introducing artificial spectral values, e.g., Fig. 4(c).

In practice, we can observe small displacements between spectral images of the same acquisition. Those might be caused by movements of experimenter hand, volunteer facial expression changes or vibrations of the filter wheel during the $2.5 \mathrm{~s}$ acquisition. We compensate these shifts by computing a non-rigid registration vector field 
between all pairs of successive spectral images. Vector fields are then composed and applied in a way that all spectral images are registered to the same reference spectral image, e.g., Fig.4(d).

The last preprocessing step is dedicated to deal with skin hairiness as it is not a component we want to take into account for the foundation make-up study. We designed a Digital Hair Removing (DHR) algorithm whose first part is to detect pixels belonging to hairiness using a linear opening and a binary thresholding. Second part is an inpainting method (Fast Marching inpainting method described in [6]) to fill in pixels classified as hairiness with values from their neighborhoods, e.g., Fig.4(e),

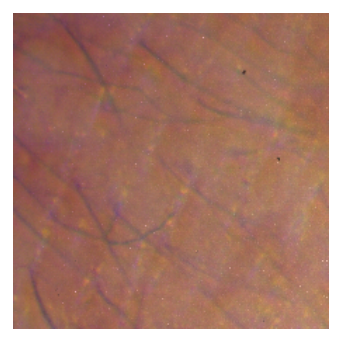

(a) Raw image sample

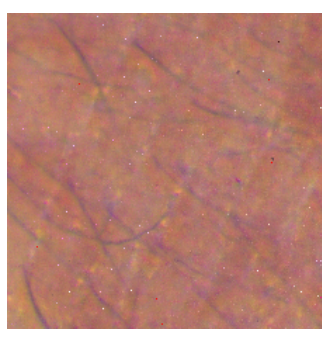

(b) Normalization

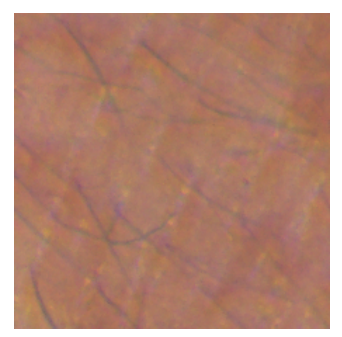

(c) Denoising

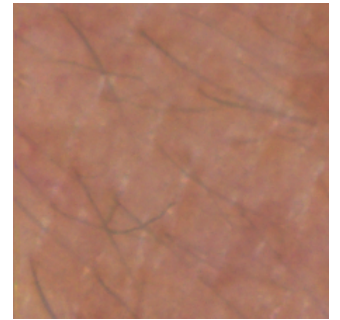

(d) Registration

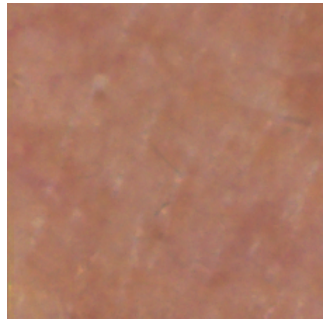

(e) Hair removing

Fig. 4. Color-rendering of a multispectral skin image after successive preprocessing steps.

\section{Dimensionality reduction by IP-PCA}

In our set of acquisitions, we noticed important redundancy level between spectral images which is a recurrent issue of multispectral imaging. Principal Component Analysis (PCA) is classically used to reduce image dimensionality since this analysis method is canonical [3|5|20|28]35] and was proved to be the most effective in the case of real application datasets [33]. Given a multispectral image, PCA computes a reduced space of representation composed of the image eigenvectors. It allows us to transform a $10 \mathrm{com}-$ ponents multispectral image into 4 eigenimages containing at least $95 \%$ of the original 
image variance.

However, PCA is only suitable for single group study, in our case, for a single multispectral image. Applying PCA to each multispectral skin image separately will generate several reduced spaces non homogeneous bases to one another because of possible inversions and permutations of eigenvectors and consequently, eigenimages which are not consistent between different acquisitions. Thus, we practice Inverted and Permuted Principal Component Analysis (IP-PCA) [9] to generate group-wise reduced spaces corrected by means of inversions and permutations as shown in Fig. 5. The algorithm detailed in [8]9], was proved to be useful on our dataset [8].

Now that redundancy is decreased, we can focus on extracting significant texture parameters on resulting eigenimages.

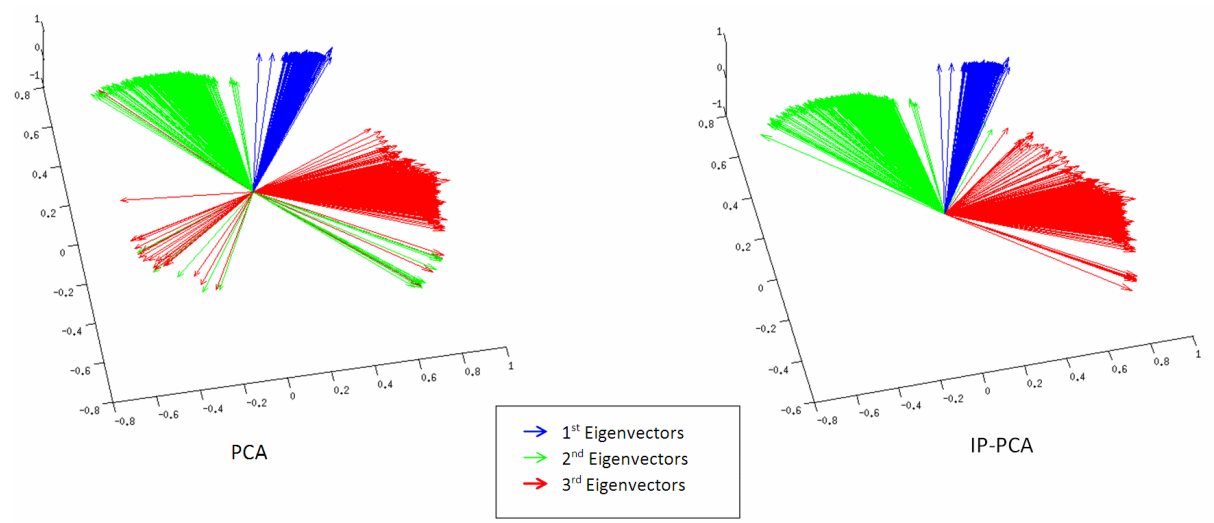

Fig. 5. Spatial representation (for the first three coordinates) of the eigenvectors of rank $\{1,2,3\}$ for the entire images base in the case of PCA (left side) and IP-PCA (right side). Each base of 3 eigenvectors corresponds to one of the multispectral images.

\section{Texture extraction from multispectral images}

Starting from our multispectral dataset, the goal is to quantify the presence of foundation make-up. This section explains the strategy of texture extraction directly on multispectral data (integrative approach). This objective can be driven following either a marginal approach, i.e., component by component, or a multivariate one using all components simultaneously. 


\subsection{Marginal approach from eigenimages}

Marginal approach has the advantage of using classical grey level algorithms on each image component. In our case, marginal texture features are extracted separately on the 4 first IP-PCA eigenimages, which are linear combinations of initial spectral images. In this sense, even marginally computed texture data take all spectral images into account. Thus, texture descriptors obtained that way can be qualified as multispectral texture descriptors.

Several texture parameters are considered in order to enrich the skin surface analysis. In the following definitions, we consider a multispectral image $F(x)=\left\{f_{1}(x), \ldots, f_{D}(x)\right\}$ composed of $D$ components defined for each pixel $x$.

\section{- Statistical moments}

Statistical moments until the $4^{\text {th }}$ order (i,e., the mean, variance, asymmetry coefficient and kurtosis) are computed from the gray level distribution.

\section{- Variogram}

The geometric variogram function [22] is a morphological texture description defined for a given angle $\theta$ and a distance $h$ by :

$$
\gamma_{f, \theta}(h)=\frac{1}{2} \operatorname{Var}\left(f(x)-f\left(x+h_{\theta}\right)\right),
$$

where $x+h_{\theta}$ is the translated pixel from $x$ at the amplitude $h$ in the direction $\theta$. The variogram itself is not used as a texture parameter. Instead some characteristic values measured on it, for instance, the range, the practical range, the slope [7]. In practice, we studied 6 parameters from variograms in 2 directions $\theta \in\left\{0^{\circ}, 90^{\circ}\right\}$.

\section{- Granulometry}

A morphological granulometry is the study on an image size distribution [23]29]. Pattern spectrum $(P S)$ is a granulometric curve obtained by measuring the difference between successive size growing morphological openings or closings. The granulometric $P S$ function $\left(P S^{+}\right)$and the anti-granulometric $P S$ function $\left(P S^{-}\right)$ are just defined by the two following equations:

$$
P S^{+}(f, n)=\frac{m\left(\gamma_{n}(f)\right)-m\left(\gamma_{n+1}(f)\right)}{m(f)},
$$

and

$$
P S^{-}(f,-n)=\frac{m\left(\varphi_{n}(f)\right)-m\left(\varphi_{n-1}(f)\right)}{m(f)},
$$

where $m$ is the Lebesgue integral of a gray level image, i.e., the sum of its pixels value, $\gamma_{n}(f)$ is the morphological opening of $f$ by the $n^{\text {th }}$ element of a size increasing family of structuring elements and $\varphi_{n}(f)$ is the dual morphological closing. Finally, the mean, variance, asymmetry coefficient and kurtosis of the $\mathrm{PS}^{+}$and $\mathrm{PS}^{-}$ functions are computed as texture parameters. 


\section{- Haralick's parameters}

Gray level co-occurrence matrices into 4 directions $\left(0^{\circ}, 45^{\circ}, 90^{\circ}, 135^{\circ}\right)$ are also computed. Among the 14 Haralick's parameters [14] we extracted the 6 parameters considered to be the less correlated [4], i.e., the energy, contrast, entropy, correlation, homogeneity and variance.

\subsection{Multivariate approach from multispectral images}

Multivariate approach takes into account all wavelengths at the same time and is practiced on original images space (instead of IP-PCA reduced spaces). A spectral distance is necessary to build texture descriptors. We selected the Spectral Angle Mapper (SAM) and the Euclidian distance because they were proved to be the most effective with our dataset [8]. For two spectra $F(x)=\left\{f_{1}(x), \ldots, f_{D}(x)\right\}$ and $F(y)=\left\{f_{1}(y), \ldots, f_{D}(y)\right\}$, the distance $\operatorname{SAM}(F(x), F(y))$ is given by:

$$
\operatorname{SAM}(F(x), F(y))=\cos ^{-1}\left(\frac{\sum_{d=1}^{D} f_{d}(x) f_{d}(y)}{\|F(x)\| .\|F(y)\|}\right) .
$$

Once the spectral distance is defined, we can extend some marginal texture definitions to the multivariate case as follows.

\section{- Multispectral variogram}

A multispectral variogram function can be obtained by generalizing the solution proposed in [21], which consists in replacing in (11) the variance operator by a spectral distance :

$$
\gamma_{F, \theta}^{D i s t}(h)=\frac{1}{2} \sum_{x} \operatorname{Dist}^{2}(F(x), F(x+h)),
$$

where Dist is a spectral distance (SAM or Euclidian in our case), cumulated on all the pixels of the image. Likewise, the same parameters are measured on the multispectral variogram curve.

\section{- Multispectral granulometry}

In order to set up a multispectral pattern spectrum function, we first rely on the color top-hat [13], to propose a multispectral circular top-hat associated to a spectral distance:

$$
\rho_{B}(F(x))=\sup _{y \in B_{x}}\left\{-\xi_{B}(y)\right\}
$$

where $B_{x}$ is the structuring element centered on $x$ and

$$
\xi_{B}(y)=\inf _{z \in B_{y}}\{-\operatorname{Dist}(F(y), F(z))\} .
$$

Then, the proposed multispectral circular Pattern Spectrum PS corresponds to 2 where $\gamma_{n}(f)$ is replaced by $\rho_{n}(F)$. Contrary to an usual top-hat, the $\rho$ has no interpretation in the sense of positive peak extraction or image order. Hence, there is no need to define an anti-granulometric function. 


\section{- Texture differential image}

To extract statistical moments and Haralick's parameters in the context of a multivariate integrative approach, we transform the multispectral initial images into texture differential images in which each pixel (of D components) is replaced by the mean distance between the pixel spectrum and spectra of the pixel neighborhood. Haralick's parameters and statistical moments are extracted afterwards on the differential image.

\section{Applications to a cosmetology study}

The protocol (see Sect.2) to study foundation make-up presence on skin with the Asclepios System mentioned that 4 acquisitions were taken on each volunteer corresponding to 4 states of the applied product (before and after foundation make-up application, 3 hours and 6 hours after application). Our goal is to predict the acquisition time of each image using its texture descriptors as predictive variables. We expect this multiclass prediction case to be able to illustrate the relevance of texture data depending on whether they are extracted marginally or in a multivariate way.

\subsection{Prediction process}

Instead of using Asclepios System entire images, texture descriptors are extracted on 25 smaller samples of initial surface. This method is called bootstraping and allows us to artificially grow the number of experiments from 720 to 180000 . Samples dimension $(256 \times 256$ pixels $)$ is chosen larger than 4 times the size of estimated biggest skin structures.

In order to process the 4 times prediction, Support Vector Machines (SVM) [32] with a Radial Basis Function (RBF) kernel and a constant $\sigma$ automaticaly defined (by the hill climbing algorithm) are used. SVM were selected among several predictors (Random Forest, Naives Bayes Classifier, linear regression,... ) because they provided the best results. Their extension to multiclass prediction is handled by the One-vs-All model. Precision score i.e. rate of correct class attribution is the considered performance criterium. It is estimated using a cross validation method compatible with the bootstrapping: the 30 volunteers are splitted into 5 groups of 6 , then data issued from a group of 6 volunteers (i.e. $20 \%$ of the available data) are iteratively used as test samples whereas data from the other groups serve for learning.

Since marginal approach produces more texture features than multivariate approach, we limit the number of multivariate features using a backward feature elimination routine (belonging to the wrapper methods) [11]. Likewise, when blending both marginal and multivariate texture features to proceed the time prediction, the same feature elimination process is used.

\subsection{Prediction results}

Prediction accuracy obtained with different texture parameters and approaches of extraction (marginal or multivariate) is given in Table 1 We can notice that the marginal 
integrative approach seems more effective. However, mixing both multivariate and marginal approaches provides the best precision rates. Thus, multivariate parameters are also interesting in our task of prediction.

Table 1. Precision scores of acquisition time prediction obtained with different texture features computed with marginal (Marg.) and multivariate (Multi.) approaches.

\begin{tabular}{lccc}
\hline Parameters & Marg. & Multi. & Marg.+Multi. \\
\hline Statistical moments (8 parameters) & $58.4 \%$ & $42.7 \%$ & $67.1 \%$ \\
Variogram (18 parmeters) & $61.9 \%$ & $50.1 \%$ & $61.9 \%$ \\
Granulometry (32 parameters) & $61.7 \%$ & $61.4 \%$ & $69.2 \%$ \\
Haralick (12 parameters) & $61.9 \%$ & $56.7 \%$ & $76.0 \%$ \\
All parameters (70 parameters) & $82.2 \%$ & $72.1 \%$ & $84.8 \%$ \\
\hline
\end{tabular}

\section{Conclusion}

This paper presented new multispectral texture features based on mathematical morphology as well as two alternatives of multispectral images texture analysis. The first is to reduce images dimensionality before measuring standard parameters on each eigenimage separately, the other is to process multivariate texture extraction directly on all the image wavelengths.

After a dedicated preprocessing chain, data from our study of foundation make-up on skin allow us to demonstrate the efficiency of both approaches and simoultaneously the validity of the new multivariate texture features.

The next step is to confirm our methods efficiency on another dataset from a different context.

\section{References}

1. Arvis, V., Debain, C., Berducat, M., Benassi, A.: Generalization of the cooccurrence matrix for colour images: application to colour texture classification. Image Analysis \& Stereology 23(1), 63-72 (2011)

2. Astola, J., Haavisto, P., Neuvo, Y.: Vector median filters. Proceedings of the IEEE 78(4), 678-689 (1990)

3. Avena, G., Ricotta, C., Volpe, F.: The influence of principal component analysis on the spatial structure of a multispectral dataset. International Journal of Remote Sensing 20(17), 33673376 (1999)

4. Baraldi, A., Parmiggiani, F.: An investigation of the textural characteristics associated with gray level cooccurrence matrix statistical parameters. IEEE Transactions on Geoscience and Remote Sensing 33(2) (1995) 
5. Baronti, S., Casini, A., Lotti, F., Porcinai, S.: Principal component analysis of visible and near-infrared multispectral images of works of art. Chemometrics and Intelligent Laboratory Systems 39(1), 103 - 114 (1997)

6. Bertalmio, M., Sapiro, G., Caselles, V., Ballester, C.: Image inpainting. In: Proceedings of the 27th annual conference on Computer graphics and interactive techniques. pp. 417-424. ACM Press/Addison-Wesley Publishing Co. (2000)

7. Bohling, G.: Introduction to geostatistics and variogram analysis. Kansas geological survey pp. 1-20 (2005)

8. Corvo, J.: Characterization of cosmetologic data from multispectral skin images. Phd thesis, Ecole Nationale Supérieure des Mines de Paris (2016)

9. Corvo, J., Angulo, J., Breugnot, J., Borbes, S., Closs, B.: Common reduced spaces of representation applied to multispectral texture analysis in cosmetology. In: SPIE BiOS. pp. 970104-970104. International Society for Optics and Photonics (2016)

10. Drimbarean, A., Whelan, P.F.: Experiments in colour texture analysis. Pattern recognition letters 22(10), 1161-1167 (2001)

11. Guyon, I., Elisseeff, A.: An introduction to variable and feature selection. Journal of machine learning research 3, 1157-1182 (2003)

12. Hanbury, A., Kandaswamy, U., Adjeroh, D.A.: Illumination-invariant morphological texture classification. In: Mathematical Morphology: 40 Years On, pp. 377-386. Springer (2005)

13. Hanbury, A.G., Serra, J.: Morphological operators on the unit circle. IEEE Transactions on Image Processing 10(12), 1842-1850 (2001)

14. Haralick, R.M., Shanmugam, K., Dinstein, I.H.: Textural features for image classification. Systems, Man and Cybernetics, IEEE Transactions on (6), 610-621 (1973)

15. Healey, G., Wang, L.: Illumination-invariant recognition of texture in color images. JOSA A 12(9), 1877-1883 (1995)

16. Jain, A., Healey, G.: A multiscale representation including opponent color features for texture recognition. IEEE Transactions on Image Processing 7(1), 124-128 (1998)

17. Jolivot, R.: Dévéloppement d'un outil d'imagerie dédié a l'acquisition, l'analyse et a la caractérisation multispectrale des lésions dermatologiques. Ph.D. thesis, Le2i laboratory, Universite de Bourgogne (2011)

18. Jolivot, R., Benezeth, Y., Marzani, F.: Skin parameter map retrieval from a dedicated multispectral imaging system applied to dermatology/cosmetology. International Journal of Biomedical Imaging (2013)

19. Kukkonen, S., Kälviäinen, H., Parkkinen, J.: Color features for quality control in ceramic tile industry. Optical Engineering 40(2), 170-177 (2001)

20. Lanir, J., Maltz, M., Rotman, S.R.: Comparing multispectral image fusion methods for a target detection task. Optical Engineering 46(6), 066402-066402 (2007)

21. Li, P., Cheng, T., Guo, J.: Multivariate image texture by multivariate variogram for multispectral image classification. Photogrammetric Engineering \& Remote Sensing 75(2), 147-157 (2009)

22. Matheron, G.: Principles of geostatistics. Economic geology 58(8), 1246-1266 (1963)

23. Matheron, G.: Eléments pour une théorie des milieux poreux. Masson (1967)

24. Messer, K., Kittler, J.: A region-based image database system using colour and texture. Pattern Recognition Letters 20(11), 1323-1330 (1999)

25. Palm, C.: Color texture classification by integrative co-occurrence matrices. Pattern Recognition 37(5), 965 - 976 (2004)

26. Palm, C., Keysers, D., Lehmann, T., Spitzer, K.: Gabor filtering of complex hue/saturation images for color texture classification. In: Int. Conf. on Computer Vision. vol. 2, pp. 45-49 (2000) 
27. Paola, J., Schowengerdt, R.: A review and analysis of backpropagation neural networks for classification of remotely-sensed multi-spectral imagery. International Journal of remote sensing 16(16), 3033-3058 (1995)

28. Paquit, V.C., Tobin, K.W., Price, J.R., Mériaudeau, F.: 3d and multispectral imaging for subcutaneous veins detection. Optics express 17(14), 11360-11365 (2009)

29. Serra, J.: Image analysis and mathematical morphology, v. 1. Academic press (1982)

30. Song, K.Y., Kittler, J., Petrou, M.: Defect detection in random colour textures. Image and vision computing 14(9), 667-683 (1996)

31. Suen, P.H., Healey, G.: Modeling and classifying color textures using random fields in a random environment. Pattern Recognition 32(6), 1009-1017 (1999)

32. Suykens, J.A., Vandewalle, J.: Least squares support vector machine classifiers. Neural processing letters 9(3), 293-300 (1999)

33. Van Der Maaten, L., Postma, E., Van den Herik, J.: Dimensionality reduction: a comparative. J Mach Learn Res 10, 66-71 (2009)

34. Van de Wouwer, G., Scheunders, P., Livens, S., Van Dyck, D.: Wavelet correlation signatures for color texture characterization. Pattern recognition 32(3), 443-451 (1999)

35. Yamaguchi, M., Mitsui, M., Murakami, Y., Fukuda, H., Ohyama, N., Kubota, Y.: Multispectral color imaging for dermatology: application in inflammatory and immunologic diseases. In: Color and Imaging Conference. vol. 2005, pp. 52-58. Society for Imaging Science and Technology (2005) 